\title{
Self-Assembling Protein Nanoparticles: implications for HIV-1 vaccine development
}

\author{
Christopher P Karch ${ }^{1,2}$, Gary R Matyas ${ }^{1}$, Peter Burkhard ${ }^{3}$ \& Zoltan Beck*,1,2 \\ ${ }^{1}$ US Military HIV Research Program, Laboratory of Adjuvant and Antigen Research, Walter Reed Army Institute of Research, 503 \\ Robert Grant Ave, Silver Spring, MD 20910, USA \\ ${ }^{2}$ Henry M Jackson Foundation for the Advancement of Military Medicine, 6720A Rockledge Drive, Bethesda, MD 20817, USA \\ ${ }^{3}$ Alpha-O Peptides, Lörracherstrasse 50, 4125 Riehen, Switzerland \\ *Author for correspondence: Tel.: +1 301319 2290: zbeck@hivresearch.org
}

\begin{abstract}
"This technology could be adapted for HIV-1 vaccine development to present the V1V2 loop in a native-like conformation to induce high titers of binding, neutralizing and functional Abs, T-cell responses targeting multiple epitopes and subdominant epitopes, all of these will hopefully expand upon and improve the results of RV144."
\end{abstract}

First draft submitted: 28 June 2018; Accepted for publication: 29 June 2018; Published online: 1 October 2018

Keywords: antigen density $\bullet$ conformational antibody $\bullet$ HIV-1 $\bullet$ nanoparticle $\bullet$ SAPN $\bullet$ trimeric epitope $\bullet$ vaccine

Self-Assembling Protein Nanoparticles: a modern platform to increase the immunogenicity of vaccines

Subunit vaccines are one of the major focuses of modern vaccine development. They tend to be more tolerable than whole organism vaccines, however, they also tend to be less immunogenic. To address this problem, different approaches such as delivery of the antigen on a particulate carrier, displaying multiple copies of an antigen and/or use of a modern adjuvant system can lead to stronger immune responses [1]. Nanoparticles can be designed to combine all of these approaches allowing for the development of effective vaccines against various pathogens [2].

One such nanoparticle platform, the Self-Assembling Protein Nanoparticles (SAPNs), have been used to develop vaccines for different infectious diseases [3-13]. SAPNs are designed using coiled-coil protein folding motifs. Each SAPN monomer is composed of two different oligomerization domains connected by a short linker. On either or both termini of these protein chains antigens can be engineered to be surface exposed on the refolded particle [14]. To increase cellular immune responses, $\mathrm{CD}^{+} \mathrm{T}_{\mathrm{H}}$ and $\mathrm{CD}^{+}$epitopes can also be engineered into both the surface exposed and core regions of the SAPNs [3-5]. Monomers spontaneously self-assemble into a three-dimensional particle that can be decorated with multiple copies of the antigens [14].

Modern vaccines contain adjuvant formulations that can enhance, modulate and prolong the immune response. SAPNs can be used in combination with a built-in adjuvant system [5,6], a traditional adjuvant [7,8] or a modern adjuvant system [9]. Thus, SAPN-based vaccines may increase not only the magnitude, but also the durability of the immune responses.

\section{Previous SAPN applications}

Malaria

The first application of the SAPN technology was the development of a malaria vaccine. The initial candidate targeted the asexual pre-erythocytic stage of the disease by presenting 60 copies of the immunodominant repeat region of the circumsporozoite protein antigen (CSP) from rodent malaria species Plasmodium berghei. This vaccine resulted in long-lasting protection in multiple mouse strains without the presence of an adjuvant [10]. A more advanced candidate for the human malaria species Plasmodium falciparum was further developed that also included the immunodominant repeat region along with the addition of multiple CSP CD8 ${ }^{+}$epitopes. It induced high antibody $(\mathrm{Ab})$ titers that again led to long lasting protection in a murine model without the addition of an adjuvant [3]. The current vaccine candidate (FMP014) included the thrombospondin Type-1 repeat ( $\alpha$ TSR) 
domains of the CSP protein, as well as multiple $\mathrm{CD}^{+}$epitopes to the core. This candidate was combined with two different adjuvant systems, admixed liposome adjuvants, Army Liposome Formulations (ALF) [9] or a built in TLR5 agonist adjuvant, flagellin [6]. Immunization of C57BL/6 mice resulted in very high immunoglobulin G (IgG) Ab titers and T-cell responses. FMP014 adjuvanted with ALF containing QS-21 (ALFQ) has been used in rhesus macaques and will be used in a controlled human malaria infection Phase I/IIa clinical trial.

SAPNs were also used to target the asexual blood stage of the parasite's life cycle with a recently identified antigen, Tex1. Two previously established immunogenic regions of the Tex1, P27 and P27A were engineered into the particle. P27A is predicated to be an intrinsically unstructured region of the protein, however, P27 is a trimeric coiled-coil. The SAPN allowed P27 to be presented in its native trimeric conformation, which led to an increase in immunogenicity in mice. The P27/P27A-SAPN reacted with sera from individuals from a malaria endemic region and these novel SAPNs are predicted to provide cross-protection between P. falciparum and Plasmodium vivax [4].

\section{SARS}

One of the other initial attempts to develop a SAPN based vaccine targeted the coronavirus causing severe acute respiratory syndrome. This SAPN presented 60 copies of the SARS B cell epitope from the C-terminal heptad repeat of the virus' spike protein. Using the SAPN scaffold, the vaccine was capable of mimicking the native trimeric coiled-coil structure of the spike. Immunization of BALB/c mice with this SAPN candidate resulted in the development of high titers of conformation specific Abs that were capable of neutralizing the SARS virus in vitro [11].

\section{Toxoplasmosis}

The primary effector response against the causative parasite Toxoplasma gondii is mediated by cytotoxic Tlymphocytes necessitating a vaccine candidate that utilizes $\mathrm{CD}^{+}$and $\mathrm{CD}^{+}$epitopes. Initial designs focused on a single antigen (dense granule 7 [GRA7 ${ }_{20-28}$ LPQATTAAT]) presented on a SAPN that contained the universal $\mathrm{CD}^{+}$epitope PADRE. This candidate was capable of eliciting antigen-specific $\mathrm{CD} 8^{+}$responses in a transgenic mouse strain for human HLA challenge model and significantly reduced $T$. gondii cysts in the brain [12]. A more advanced candidate was designed to contain $\mathrm{CD}^{+}$epitopes from the surface antigen-1 (SAG1), the dense granule proteins (GRA5 and GRA6), and the SAG1-related sequences (SRS52A). In addition, this candidate with a built in flagellin adjuvant was admixed the TLR 4 agonist GLA-SE. The construct also contained multiple universal CD $4^{+}$ epitopes. As predicted, this vaccine elicited specific $\mathrm{CD}^{+}$responses and it significantly reduced the T. gondii cysts in the mouse brain [7].

\section{Influenza}

The first attempt at a universal influenza A vaccine utilized the external domains of the viral membrane proton channel M2. By presenting 48 copies of this antigen in its native tetrameric conformation on a SAPN, vaccinated animals developed high $\mathrm{Ab}$ titers that were capable of reducing viral shedding during a low pathogenic viral challenge in chickens [13]. A more advanced construct contained not only M2, but also the conserved Helix C of the hemagglutinin stalk, which was presented it in its native trimeric conformation. This candidate also had multiple universal $\mathrm{CD}^{+}$epitopes as well as the immunostimulatory molecule flagellin. Higher titers of Abs were obtained in animals immunized with self-adjuvanted SAPNs than nonadjuvanted constructs. Vaccinated chickens were capable of raising Abs with neutralizing activity against multiple viral subtypes. In a mouse model, animals were completely protected from a high pathogenic influenza challenge [5].

\section{SAPNs for HIV-1 vaccine development}

A SAPN-based vaccine may be optimal for the development of a potent HIV-1 vaccine. Previously, we developed a SAPN that contained the membrane proximal external region antigen (MPER) of HIV-1. This candidate was capable of inducing high Ab titers, however, not surprisingly, those Abs were not neutralizing [8]. To date, MPERbased vaccine candidates have been able to generate neutralizing Abs with only low potency and breadth [15]. Recently, a new target was identified in a Phase III clinical trial, RV144. In this trial, modest efficacy against HIV-1 acquisition was demonstrated, and the risk of infection was inversely correlated with the presence IgG Abs raised against the V1V2 loop of the virus envelope protein [16,17].

The V1V2 loop represented only a small fraction of the RV144 immunogens in the vaccination regimen, and a subunit vaccine with increased copies of this region may lead to a better vaccine. To develop a strong Ab response 
Figure 1 Computer models of a potential V1V2 Self-Assembling Protein Nanoparticles. The V1V2 loop is highlighted. (A) SAPN monomer design. (B) Side view of the V1V2 trimer on the coiled-coil arm of SAPN. Three SAPN-monomers assembled as a trimer. (C) Top-down view of the V1V2 trimer. (D) The fully assembled SAPN structure. Icosahedral SAPNs display 20 copies of the V1V2 trimer. Color scheme: Grey: HisTag; Green: Pentamer; Blue: Trimer; Red: Stem of the V1V2 loop; Gold: V1 loop; Purple: V2 loop.

SAPN: Self-assembling protein nanoparticle.

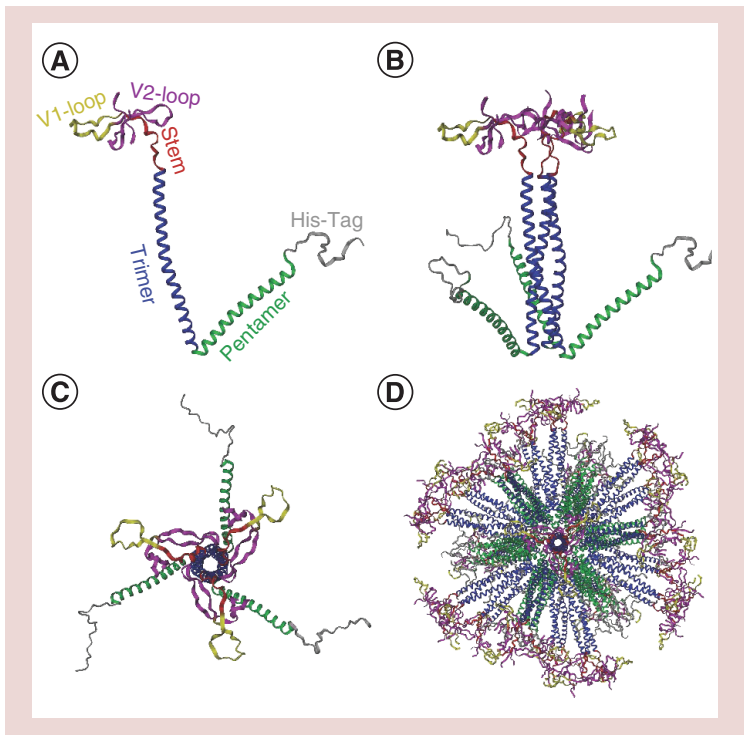

to viral surface proteins it is known that the spacing and density of these epitopes is important [18]. SAPNs allow for high density presentation of the V1V2 loop and optimal spacing to increase the Ab response. It is also believed that the trimeric presentation of the V1V2 loop is essential for the development of functional Abs [19]. The spontaneous formation of the trimeric coiled-coils of the SAPN allows 20 copies of the V1V2 loop trimer to be presented. By using computer modeling the SAPNs can be optimally designed, so the V1V2 loop can be presented in a native-like trimeric conformation (Figure 1).

An effective vaccine needs to induce functional Abs. Previous studies have indicated that antibodies raised against the V1V2 loop do not function through neutralization, but through other antibody-mediated mechanisms such as antibody-dependent cell-mediated cytotoxicity (ADCC), antibody-dependent cell-mediated phagocytosis (ADCP), and antibody-dependent complement-mediated lysis (ADCML) [20-22]. By incorporating universal $\mathrm{CD}^{+}{ }^{+}$epitopes into the SAPN core there should be an increase in the titer of functional Abs to the V1V2 loop as demonstrated with previous SAPN candidates [3,5,7]. Due to the native-like presentation of the V1V2 loop, conformational Abs should be generated, again increasing the functionality of the vaccine.

A major concern with HIV-1 vaccine development is the sequence diversity of the virus. We can address this problem by two different approaches. The first is the choice of the V1V2 loop sequence that is presented on the SAPNs. By identifying loops that contain reduced hypervariable regions we can direct the immune response to the more conserved residues. We have previously demonstrated that the influenza candidates containing highly conserved sequences can result in a vaccine candidate that is cross-reactive between different strains [5]. Another approach is to generate particles that are co-assembled from SAPN monomers that contain different V1V2 loops. A tailored vaccine candidate can be developed for each geographical region taking into account the predominantly circulating strains.

Despite the failure of T-cell vaccines tested to date, cellular immunity may be needed for an efficacious prophylactic vaccine. Non-human primate (NHP) studies support the potential efficacy of a prophylactic T-cell vaccine [23]. In addition to the $\mathrm{B}$ cell immunogen, SAPNs can contain multiple $\mathrm{CD}^{+}{ }^{+}$and $\mathrm{CD}^{+}$epitopes in their cores and universal and/or HIV-1-specific $\mathrm{CD}^{+}$sequences that may induce a balanced humoral and cellular immune response to the vaccine.

\section{Conclusion}

SAPNs are a unique vaccine platform. They can display B cell immunogens, are capable of multivalent presentation of epitopes and have the potential to induce $\mathrm{CD} 8^{+} \mathrm{T}$-cell responses through cross presentation. The immune response to a SAPN-based vaccine can further be increased by the incorporation of the TLR5-agonist flagellin into the particle [5,6] or by the addition of potent next generation adjuvants such as one of the Army Liposome Formulations [24]. This technology could be adapted for HIV-1 vaccine development to present the V1V2 loop in a native-like conformation to induce high titers of binding, neutralizing and functional Abs, T-cell responses 
targeting multiple epitopes and subdominant epitopes, all of these will hopefully expand upon and improve the results of RV144. SAPNs also allow for reasonably low cost and high yield production of the V1V2 loop that provide a very promising platform for HIV-1 vaccine development.

Financial \& competing interest disclosure

The views expressed are those of the authors and should not be construed to represent the positions of the US Army or the Department of Defense. This work was supported by a cooperative agreement (W81XWH-11-2-0174) between the Henry M Jackson Foundation for the Advancement of Military Medicine, Inc., and the US Department of Defense.

Peter Burkhard has an interest in the company Alpha-O Peptides that has patents or patents pending on the technology.

The authors have no other relevant affiliations or financial involvement with any organization or entity with a financial interest in or financial conflict with the subject matter or materials discussed in the manuscript apart from those disclosed.

No writing assistance was utilized in the production of this manuscript.

\section{Open access}

This work is licensed under the Attribution-NonCommercial-NoDerivatives 4.0 Unported License. To view a copy of this license, visit http://creativecommons.org/licenses/by-nc-nd/4.0/

\section{References}

1. Karch CP, Burkhard P. Vaccine technologies: from whole organisms to rationally designed protein assemblies. Biochem. Pharmacol. 120, 1-14 (2016).

2. Boraschi D, Italiani P. From antigen delivery system to adjuvanticy: the board application of nanoparticles in vaccinology. Vaccines (Basel) 3(4), 930-939 (2015).

3. Kaba SA, McCoy ME, Doll TA et al. Protective antibody and CD8 ${ }^{+}$T-cell responses to the Plasmodium falciparum circumsporozoite protein induced by a nanoparticle vaccine. PLoS ONE 7(10), e48304 (2012).

4. Karch CP, Doll T, Paulillo SM et al. The use of a $P$. falciparum specific coiled-coil domain to construct a self-assembling protein nanoparticle vaccine to prevent malaria. J. Nanobiotechnol. 15(1), 62 (2017).

5. Karch CP, Li J, Kulangara C et al. Vaccination with self-adjuvanted protein nanoparticles provides protection against lethal influenza challenge. Nanomedicine 13(1), 241-251 (2017).

6. Kaba SA, Karch CP, Seth L et al. Self-Assembling Protein Nanoparticles with built-in flagellin domains increases protective efficacy of a Plasmodium falciparum based vaccine. Vaccine 36(6), 906-914 (2018).

7. El Bissati K, Zhou Y, Paulillo SM et al. Protein nanovaccine confers robust immunity against Toxoplasma. NPJ Vaccines 2, 24 (2017).

8. Wahome N, Pfeiffer T, Ambiel I et al. Conformation-specific display of 4E10 and 2F5 epitopes on Self-Assembling Protein Nanoparticles as a potential HIV vaccine. Chem. Biol. Drug Des. 80(3), 349-357 (2012).

9. Seth L, Bingham Ferlez KM, Kaba SA et al. Development of a self-assembling protein nanoparticle vaccine targeting Plasmodium falciparum circumsporozoite protein delivered in three army liposome formulation adjuvants. Vaccine 35(41), 5448-5454 (2017).

10. Kaba SA, Brando C, Guo Q et al. A nonadjuvanted polypeptide nanoparticle vaccine confers long-lasting protection against rodent malaria. J. Immunol. 183(11), 7268-7277 (2009).

11. Pimentel TA, Yan Z, Jeffers SA, Holmes KV, Hodges RS, Burkhard P. Peptide nanoparticles as novel immunogens: design and analysis of a prototypic severe acute respiratory syndrome vaccine. Chem. Biol. Drug Des. 73(1), 53-61 (2009).

12. El Bissati K, Zhou Y, Dasgupta D et al. Effectiveness of a novel immunogenic nanoparticle platform for Toxoplasma peptide vaccine in HLA transgenic mice. Vaccine 32(26), 3243-3248 (2014).

13. Babapoor S, Neef T, Mittelholzer C et al. A novel vaccine using nanoparticle platform to present immunogenic M2e against avian influenza infection. Influenza Res. Treat. 2011, 126794 (2011).

14. Raman S, Machaidze G, Lustig A, Aebi U, Burkhard P. Structure-based design of peptides that self-assemble into regular polyhedral nanoparticles. Nanomedicine 2(2), 95-102 (2006).

15. Liu H, Su X, Si L, Lu L, Jiang S. The development of HIV vaccines targeting gp 41 membrane-proximal external region (MPER): challenges and prospects. Protein Cell doi:10.1007/s13238-018-0534-7 (2018). (Epub ahead of print)

16. Haynes BF, Gilbert PB, Mcelrath MJ et al. Immune-correlates analysis of an HIV-1 vaccine efficacy trial. N. Engl. J. Med. 366(14), 1275-1286 (2012).

17. Rerks-Ngarm S, Pitisuttithum P, Nitayaphan $S$ et al. Vaccination with ALVAC and AIDSVAX to prevent HIV-1 infection in Thailand. N. Engl. J. Med. 361(23), 2209-2220 (2009).

18. Schiller J, Chackerian B. Why HIV virions have low numbers of envelope spikes: implications for vaccine development. PLoS Pathog. 10(8), e1004254 (2014). 
19. O'connell RJ, Kim JH, Excler JL. The HIV-1 gp120 V1V2 loop: structure, function and importance for vaccine development. Expert Rev. Vaccines 13(12), 1489-1500 (2014).

20. Mujib S, Liu J, Rahman A et al. Comprehensive cross-clade characterization of antibody-mediated recognition, complement-mediated lysis, and cell-mediated cytotoxicity of HIV-1 envelope-specific antibodies toward eradication of the HIV-1 reservoir. J. Virol. 91(16), 1-23 (2017).

21. Musich T, Li L, Liu L, Zolla-Pazner S, Robert-Guroff M, Gorny MK. Monoclonal antibodies specific for the V2, V3, CD4-binding site, and gp 41 of HIV-1 mediate phagocytosis in a dose-dependent manner. J. Virol. 91(8), 1-13 (2017).

22. Corey L, Gilbert PB, Tomaras GD, Haynes BF, Pantaleo G, Fauci AS. Immune correlates of vaccine protection against HIV-1 acquisition. Sci. Transl. Med. 7(310), 310rv317 (2015).

23. Wijesundara DK, Ranasinghe C, Grubor-Bauk B, Gowans EJ. Emerging targets for developing T cell-mediated vaccines for human immunodeficiency virus (HIV)-1. Front. Microbiol. 8, 2091 (2017).

24. Beck Z, Matyas GR, Jalah R, Rao M, Polonis VR, Alving CR. Differential immune responses to HIV-1 envelope protein induced by liposomal adjuvant formulations containing monophosphoryl lipid A with or without QS21. Vaccine 33(42), 5578-5587 (2015). 
(

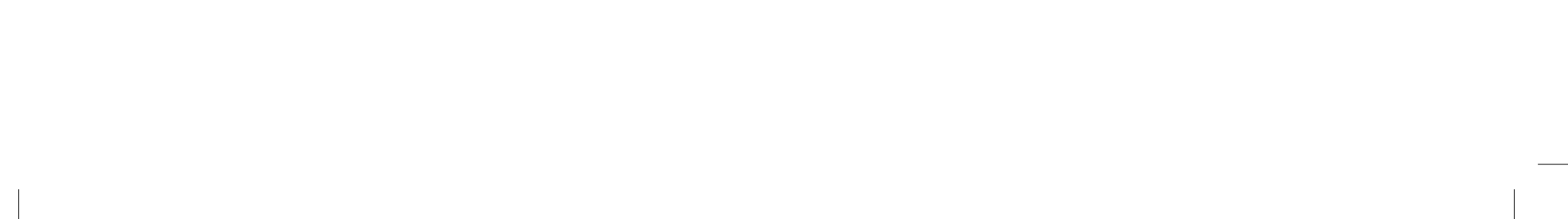

